\title{
AN ELEMENTARY PROOF OF THE GROTHENDIECK INEQUALITY
}

\author{
RON C. BLEI
}

ABSTRACT. An elementary proof of the Grothendieck inequality is given.

Since its appearance in [2, pp. 59-64] and reformulation in [3, pp. 277-280], Grothendieck's fundamental inequality has enjoyed several restatements and proofs within various frameworks of analysis (detailed accounts of which appear in [4]). The purpose of this note is to give an elementary and self-contained proof of the inequality: the argument below, an adaptation of the proof given in [1], requires knowing only that the expectation of a product of independent random variables equals the product of their expectations.

Let $\mathbf{R}^{\mathbf{N}}$ denote the space of sequences of real numbers with finitely many nonzero terms. $\mathbf{R}^{\mathbf{N}}$ will be equipped with the usual inner product,

$$
\langle x, y\rangle=\sum_{n} x(n) y(n), \quad x, y \in \mathbf{R}^{\mathbf{N}}
$$

and Euclidean norm,

$$
\|x\|=\langle x, x\rangle^{1 / 2}, \quad x \in \mathbf{R}^{\mathbf{N}} .
$$

$B$ will denote the unit ball in $\mathbf{R}^{\mathbf{N}}$, i.e. $B=\left\{x \in \mathbf{R}^{\mathbf{N}}:\|x\| \leq 1\right\}$.

THEOREM (GROTHENDIECK'S INEQUALITY). Let $\left(a_{m n}\right)_{m, n=1}^{\infty}$ be an array of complex numbers which satisfies

$$
\left|\sum_{m, n=1}^{N} a_{m n} s_{m} t_{n}\right| \leq \max _{1 \leq m, n \leq N}\left|s_{m}\right|\left|t_{n}\right|
$$

for all sequences of complex numbers $\left(s_{m}\right)_{m=1}^{\infty},\left(t_{n}\right)_{n=1}^{\infty}$, and all integers $N \geq 1$. Then, for all sequences of vectors in $\mathbf{R}^{\mathbf{N}},\left(x_{m}\right)_{m=1}^{\infty},\left(y_{n}\right)_{n=1}^{\infty}$,

$$
\left|\sum_{m, n=1}^{N} a_{m n}\left\langle x_{m}, y_{n}\right\rangle\right| \leq K \max _{1 \leq m, n \leq N}\left\|x_{m}\right\|\left\|y_{n}\right\|
$$

for all $N \geq 1$ and some universal constant $K$.

To start, define a real-valued function on $\mathbf{R}^{\mathbf{N}} \times \mathbf{R}^{\mathbf{N}}$ by

$$
A(x, y)=\prod_{n}(1+x(n) y(n)), \quad x, y \in \mathbf{R}^{\mathbf{N}}
$$

and estimate

$$
|A(x, y)| \leq e^{\sum \ln (1+|x(n) y(n)|)} \leq e^{\sum|x(n) y(n)|} \leq e^{\|x\| \cdot\|y\|} .
$$

Received by the editors January 2, 1986 and, in revised form, March 20, 1986.

1980 Mathematics Subject Classification (1985 Revision). Primary 26D15, 46C99; Secondary 47A68.

The author was partially supported by NSF Grant MCS-8301659. 
LEMMA 1. Suppose $\left(a_{m n}\right)_{m, n=1}^{\infty}$ satisfies the hypothesis of the theorem above. Then, for all sequences of vectors $\left(x_{m}\right)_{m=1}^{\infty},\left(y_{n}\right)_{n=1}^{\infty}$ in the unit ball of $\mathbf{R}^{\mathbf{N}}$,

$$
\left|\sum_{m, n=1}^{N} a_{m n} A\left(x_{m}, y_{n}\right)\right| \leq e
$$

for all $N \geq 1$.

PROOF. Let $\left(Z_{n}\right)_{n=1}^{\infty}$ be a sequence of independent real-valued random variables on some probability space so that

$$
\mathbf{E}\left(Z_{n}\right)=0, \quad \mathbf{E}\left(Z_{n}^{2}\right)=1, \quad \text { and } \quad\left|Z_{n}\right|=1 \quad \text { a.s. for all } n .
$$

(E denotes expectation; $\left(Z_{n}\right)_{n=1}^{\infty}$ could be taken as the usual system of Rademacher functions.) Given $x \in \mathbf{R}^{\mathbf{N}}$, define a random variable

$$
F(x)=\prod_{n}\left(1+i x(n) Z_{n}\right) \quad(i=\sqrt{-1}),
$$

and estimate (by (4))

$$
|F(x)| \leq\left(\prod_{n}\left(1+x(n)^{2}\right)\right)^{1 / 2} \leq e^{\|x\|^{2} / 2} \quad \text { almost surely }
$$

For any $x, y \in \mathbf{R}^{\mathbf{N}}$,

$$
\begin{aligned}
\mathbf{E}(F(x) \overline{F(y)}) & =\prod_{n} \mathbf{E}\left(1+i x(n) Z_{n}\right)\left(1-i y(n) Z_{n}\right) \quad \text { (by independence) } \\
& =\prod_{n}(1+x(n) y(n)) \quad(\text { by }(5)) \\
& =A(x, y) .
\end{aligned}
$$

Therefore, for any $\left(x_{m}\right)_{m=1}^{\infty},\left(y_{n}\right)_{n=1}^{\infty} \subset B$ and all $N \geq 1$,

$$
\begin{aligned}
\left|\sum_{m, n=1}^{N} a_{m n} A\left(x_{m}, y_{n}\right)\right| & =\left|\sum_{m, n=1}^{N} a_{m n} \mathbf{E}\left(F\left(x_{m}\right) \overline{F\left(y_{n}\right)}\right)\right| \\
& \leq \mathbf{E}\left|\sum_{m, n=1}^{N} a_{m n} F\left(x_{m}\right) \overline{F\left(y_{n}\right)}\right| \\
& \leq e \quad \text { (by (1) and (6)). Q.E.D. }
\end{aligned}
$$

Next, expand the product on the right-hand side of (3):

$$
A(x, y)=1+\langle x, y\rangle+\cdots+\sum_{n_{1}>\cdots>n_{J}} x\left(n_{1}\right) \cdots x\left(n_{J}\right) y\left(n_{1}\right) \cdots y\left(n_{J}\right)+\cdots
$$

Let $\left\{E_{J}\right\}_{J=2}^{\infty}$ be an infinite partition of the natural numbers $\mathbf{N}$, so that each $E_{J} \subset \mathbf{N}$ is infinite. Let $W_{J}$ be the $J$-dimensional wedge in $\mathbf{N}^{J}$ given by

$$
W_{J}=\left\{\left(n_{1}, \ldots, n_{J}\right) \in \mathbf{N}^{J}: n_{1}>\cdots>n_{J}\right\}
$$

and set up a one-to-one correspondence between $E_{J}$ and $W_{J}, J \geq 2$ :

$$
n \in E_{J} \leftrightarrow\left(n_{1}, \ldots, n_{J}\right) \in W_{J}
$$


Given an arbitrary $x \in B$, define a vector $\phi(x)=(\phi(x)(n))_{n \in \mathbf{N}}$ in $\mathbf{R}^{\mathbf{N}}$ by

$$
\phi(x)(n)=x\left(n_{1}\right) \cdots x\left(n_{J}\right), \quad n \in E_{J}, J=2, \ldots,
$$

and estimate

$$
\begin{aligned}
\|\phi(x)\| & =\left(\sum_{J=2}^{\infty} \sum_{n \in E_{J}}\left(x\left(n_{1}\right) \cdots x\left(n_{J}\right)\right)^{2}\right)^{1 / 2} \\
& \leq\left(\sum_{J=2}^{\infty} \frac{1}{J !}\left(\sum_{n \in \mathbf{N}}^{\infty} x(n)^{2}\right)^{J}\right)^{1 / 2} \leq(e-2)^{1 / 2} \equiv \delta<1 .
\end{aligned}
$$

Write

$$
\phi_{\delta}(x)=\phi(x) / \delta, \quad x \in B,
$$

and, by the estimate above, note that $\phi_{\delta}$ is a map from $B$ into $B$. Solving for $\langle x, y\rangle$ in (7), $x, y \in B$, we obtain

$$
\langle x, y\rangle=A(x, y)-1-\delta^{2}\left\langle\phi_{\delta}(x), \phi_{\delta}(y)\right\rangle .
$$

Therefore, applying (9) recursively, we obtain for each $J>0$

$$
\langle x, y\rangle=\sum_{j=0}^{J}\left(-\delta^{2}\right)^{j}\left[A\left(\phi_{\delta}^{j}(x), \phi_{\delta}^{j}(y)\right)-1\right]+\left(-\delta^{2}\right)^{J+1}\left\langle\phi_{\delta}^{J+1}(x), \phi_{\delta}^{J+1}(y)\right\rangle
$$

( $\phi_{\delta}^{j}$ denotes the $j$ th iterate of $\phi_{\delta}$ ). Finally, letting $J \rightarrow \infty$ in (10), we deduce

LEMMA 2. For all $x, y \in B$

$$
\langle x, y\rangle=\sum_{j=0}^{\infty}\left(-\delta^{2}\right)^{j}\left[A\left(\phi_{\delta}^{j}(x), \phi_{\delta}^{j}(y)\right)-1\right] .
$$

Proof OF GRothendieCK'S INEQUALITY. It suffices to establish (2) for $\left(x_{m}\right)_{m=1}^{\infty},\left(y_{n}\right)_{n=1}^{\infty} \subset B$. By Lemmas 1 and 2 , we estimate

$$
\begin{aligned}
\left|\sum_{m, n=1}^{N} a_{m n}\left\langle x_{m}, y_{n}\right\rangle\right| & \leq \sum_{j=0}^{\infty} \delta^{2 j}\left|\sum_{m, n=1}^{N} a_{m n}\left[A\left(\phi_{\delta}^{j}\left(x_{m}\right), \phi_{\delta}^{j}\left(y_{n}\right)\right)-1\right]\right| \\
& \leq \sum_{j=0}^{\infty} \delta^{2 j}(e+1)=(e+1) /(3-e) . \text { Q.E.D. }
\end{aligned}
$$

\section{REFERENCES}

1. R. C. Blei, A uniformity property for $\Lambda(2)$ sets and Grothendieck's inequality, Sympos. Math. 22 (1977), 321-336.

2. A. Grothendieck, Résumé de la théorie métrique des produits tensoriels topologique, Bol. Soc. Mat. São Paulo 8 (1956), 1-79.

3. J. Lindenstrauss and A. Pelczynski, Absolutely summing operators in $\mathcal{L}^{p}$-spaces and their applications, Studia Math. 29 (1968), 275-326.

4. G. Pisier, Factorization of linear operators and geometry of Banach spaces, CBMS Regional Conf. Ser. in Math. vol. 60, Amer. Math. Soc., Providence, R.I., 1986.

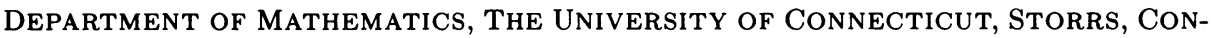
NECTICUT 06268 